\title{
NEW ITERATIVE APPROXIMATION FOR A SYSTEM OF GENERALIZED NONLINEAR VARIATIONAL INCLUSIONS WITH SET-VALUED MAPPINGS IN BANACH SPACES
}

\author{
HENG-YOU LAN, NAN-JiNG HuANG AND YeOL JE ChO
}

\begin{abstract}
In this paper, we introduce and study a new system of generalized nonlinear variational inclusions involving generalized $m$-accretive mappings. By using the resolvent operator technique for generalized $m$-accretive mapping due to Huang and Fang [10], we also prove the existence theorems of the solution and convergence theorems of the generalized Mann iterative procedures with mixed errors for this system of variational inclusions in $q$-uniformly smooth Banach spaces.
\end{abstract}

Mathematics subject classification (2000): 49J40, 47H19.

Key words and phrases: Generalized $m$-accretive mappings; a system of generalized nonlinear variational inclusions; Mann iteration with mixed errors; $q$-uniformly smooth Banach space; existence and convergence.

\section{REFERENCES}

[1] C. BAIOCCHI, A. CAPELO, Variational and Quasivariational Inequalities, Application to Free Boundary Problems, Wiley, New York, 1984.

[2] X. P. DING, Generalized quasi-variational inclusions with nonconvex functionals, Appl. Math. Comput., 122, (2001), 267-282.

[3] F. GianNeSSI, A. MAUGeRI, Variational Inequalities and Network Equilibrium Problems, Plenum, New York, 1995.

[4] A. HASSONI, A. MoudAfi, A perturbed algorithm for variational inequalities, J. Math. Anal. Appl., 185, (1994), 706-712.

[5] N. J. HUANG, Generalized nonlinear implicit quasivariational inclusion and an application to implicit variational inequalities, Z. Angew. Math. Mech., 79, (1999), 569-575.

[6] N. J. HuANG, M. R. BAI, Y. J. CHO AND S. M. KANG, Generalized nonlinear mixed quasi-variational inequalities, Comput. Math. Appl., 40, (2000), 205-215.

[7] N. J. HuAng, On the generalized implicit quasivariational inequalities, J. Math. Anal. Appl., 216, (1997), 197-210.

[8] N. J. HUANG, Mann and Ishikawa type perturbed iterative algorithms for generalized nonlinear implicit quasi-variational inclusions, Comput. Math. Appl., 35, 10 (1998), 1-7.

[9] N. J. HUANG, Y. P. FANG, A new class of general variational inclusions involving maximal $\eta$-monotone mappings, Publ. Math. Debrecen, 62, (2003), 83-98.

[10] N. J. HuANG, Y. P. FANG, Generalized $m$-accretive mappings in Banach spaces, J. Sichuan Univ., 38, 4 (2001), 591-592.

[11] N. J. HuANG, Y. P. FANG AND C. X. DENG, Nonlinear variational inclusions involving generalized $m$-accretive mappings, Proceedings of the Bellman Continuum: International Workshop on Uncertain Systems and Soft Computing, Beijing, China, July 24-27, 2002, pp. 323-327.

[12] N. J. HuANG, Nonlinear Implicit quasi-variational inclusions involving generalized $m$-accretive mappings, Arch. Inequal. Appl. , 2, 4 (2004), 413-425.

[13] K. R. KAZMI, Mann and Ishikawa type perturbed iterative algorithms for generalized quasivariational inclusions, J. Math. Anal. Appl., 209, (1997), 572-584. 
[14] L. S. LiU, Ishikawa and Mann iterative process with errors for nonlinear strongly accretive mappings in Banach spaces, J. Math. Anal. Appl., 194, (1995), 114-125.

[15] H. Z. NIE, Z. Q. LIU, K. H. KIM AND S. M. KANG, A system of nonlinear variational inequalities involving strongly monotone and pseudocontractive mappings, Adv. Nonlinear Var. Inequal., 6, 2 (2003), 91-99.

[16] S. B. NALDER, Multi-valued contraction mappings, Pacific J. Math., 30, (1969), 475-488.

[17] H. K. XU, Inequalities in Banach spaces with applications, Nonlinear Anal., 16, 12 (1991), 1127-1138.

[18] J. C. YAO, Existence of generalized variational inequalities, Oper. Res. Lett., 15, (1994), 35-40.

[19] G. X. Z. YUAN, KKM Theory and Applications, Marcel Dekker, 1999.

[20] J. X. ZHOU, G. CHEN, Digonal convexity conditions for problems in convex analysis and quasivariational inequalities, J. Math. Anal. Appl., 132, (1988), 213-225. 